\title{
Vincenzo Consolo, dal sorriso allo spasimo: l'impossibile romanzo
}

\section{Lise Bossi}

\section{(2) OpenEdition}

1 Journals

Edizione digitale

URL: https://journals.openedition.org/cher/1199

DOI: $10.4000 /$ cher.1199

ISSN: 2803-5992

\section{Editore}

Presses universitaires de Strasbourg

\section{Edizione cartacea}

Data di pubblicazione: 6 novembre 2018

Paginazione: 87-97

ISBN: 979-10-344-0027-0

ISSN: 1968-035X

Notizia bibliografica digitale

Lise Bossi, «Vincenzo Consolo, dal sorriso allo spasimo: l'impossibile romanzo», reCHERches [Online], 21 | 2018, online dal 05 octobre 2021, consultato il 21 novembre 2021. URL: http://

journals.openedition.org/cher/1199; DOl: https://doi.org/10.4000/cher.1199 


\title{
Vincenzo Consolo, dal sorriso allo spasimo: l'impossibile romanzo
}

\author{
LISE BOSSI ${ }^{1}$
}

\begin{abstract}
Aborriva il romanzo, questo genere scaduto, corrotto, impraticabile. Se mai ne aveva scritti, erano i suoi in una diversa lingua, dissonante, in una furia verbale ch'era finita in urlo, s'era dissolta nel silenzio.
\end{abstract}

(Consolo 2000: 105)

\begin{abstract}
$\mathrm{A}$ bbiamo scelto di incamminarci sulle orme di Consolo, lungo la traiettoria esistenziale e letteraria da lui percorsa, seguendo i titoli delle tre opere che costituiscono quello che egli stesso ha chiamato «Il ciclo dell'Italia unita» (De Gregorio 2013)2 ${ }^{2}$ una traiettoria che va quindi dal sorriso risorgimentale allo spasimo degli anni Novanta attraversando la notte buia del Ventennio fascista. Ci è parso ovvio infatti che, alla stregua di tutte le scelte stilistiche di Consolo, la scelta di quei titoli non fosse e non potesse essere gratuita: attraverso quei titoli ci veniva offerta una strada per arrivare fino all'ultima sua opera, Lo Spasimo di Palermo (Consolo 2000), cioè fino all'ultima tappa di un lungo e doloroso viaggio, fino agli ultimi frammenti, alle ultime parole troncate e stroncate di una conversazione nostalgica e metaforica con e sulla Sicilia.
\end{abstract}

1 Lise Bossi, Université Paris-Sorbonne.

2 «Si tratterebbe del racconto dei tre fallimenti della storia unitaria. Nel primo, sarebbe rappresentato il fallimento risorgimentale, il naufragio delle speranze democratiche e garibaldine nella Sicilia dei Gattopardi; e con esso, è descritta anche l'eclissi dell'intellettuale: inattuale, inconsapevole, passivo, non ancora engagé, per così dire. Nel secondo romanzo, Nottetempo, casa per casa, anche qui un intellettuale, maestro elementare, è travolto dal secondo e drammatico fallimento dell'Italia unitaria, quello dovuto all'avvento del fascismo. Per la seconda volta, le speranze degli italiani naufragano in un gorgo storico, nel Ventennio del buio novecentesco. E infine il terzo romanzo, Lo Spasimo di Palermo, ci presenta un ulteriore fallimento delle speranze italiane, quello repubblicano. Le speranze di una rinascita crollano di fronte alla pervasività del potere mafioso, e alle sue infiltrazioni nello Stato repubblicano». 
Allo stesso modo, non poteva essere e non è gratuito il fatto che Consolo apra Lo Spasimo di Palermo, quel libro che porta quindi il dolore iscritto nel proprio titolo, con una citazione tratta dal Prometeo incatenato di Eschilo, in cui Prometeo, colui che viene martoriato per aver portato la luce, ossia, metaforicamente e ulissianamente, virtute e conoscenza al genere umano, dichiari che «il racconto è dolore ma anche il silenzio è dolore» (Consolo 2000: 7). Questo incipit fa sorgere un certo numero di domande poiché, com’è risaputo, un incipit - e Consolo, autore di un saggio sulle Epigrafi (Consolo 1999b), più di ogni altro lo sa e lo mette in pratica - fornisce indicazioni sul significato, se non sul contenuto, dell'opera che apre.

Una domanda, per cominciare, sull'ambiguità della dichiarazione di Prometeo, prima delle tante e senz'altro volute ambiguità che troveremo nel testo di Consolo non appena si tratterà di racconto o, meglio, di narrazione. Tale dichiarazione significa infatti che il raccontare è doloroso quanto o più del tacere, oppure che, quando si tratta di esprimere il dolore, il silenzio è efficace quanto o più del racconto?

Nella prima ipotesi, l'incipit ci induce a pensare che il racconto sia una specie di male minore al quale per lo scrittore sia stato giocoforza rassegnarsi pur di non incorrere nel dolore maggiore che sarebbe potuto derivare dal silenzio. Nella seconda ipotesi, lo stesso incipit ci porta a pensare che il silenzio non sia meno adatto del racconto a dire il dolore, ma anzi, possa esserne l'espressione più alta e compiuta. Se accolta, questa seconda interpretazione implica che il dolore, lo spasimo, che, come la sciasciana linea della palma, si dilata «da Palermo, alla Sicilia, al mondo» (Consolo 2000: 112), si può dire facendo a meno del racconto. Ovvero, se proseguiamo fino in fondo con il ragionamento, ciò significa che Lo Spasimo di Palermo è un non-racconto nei cui silenzi viene detto il dolore prima dell'opera, e il dolore espresso nell'opera s'inabissa definitivamente nel silenzio. Il che, se guardiamo alla bibliografia di Consolo, corrisponde esattamente alla realtà, giacché, dopo Lo Spasimo, egli non ha effettivamente più scritto nessun racconto, romanzo o anti-romanzo.

Queste prime constatazioni ci permettono, tra l'altro, di capire perché Lo Spasimo di Palermo si configuri insieme come un testamento e un tombeau letterario. Un testamento, perché l'opera si chiude proprio con una lettera in forma di bilancio personale e intellettuale scritta dal protagonista al proprio figlio; ma anche di bilancio dall'autore al proprio lettore, prima che chi scrive scompaia con le sue ultime parole ancora nella penna, in una di quelle esplosioni al tritolo che ricorrono in un crescendo ossessivo negli ultimi racconti di Consolo, proprio come nei giorni e nelle notti di Palermo. E Lo Spasimo è anche un tombeau poetico-letterario perché, posto fin dall'inizio sotto il segno di un'oscillazione tra i due poli opposti e complementari del racconto e del silenzio, quest'ultimo opus sembra destinato a spiegare le ragioni di tale oscillazione, offrendo al lettore una specie di compendium di tutti i precedenti tentativi stilistici attraverso i quali Consolo ha cercato di proporre un epos alternativo che, partendo da una lingua poematica, lo ha portato fino all'urlo panico, per poi riportarlo alla narrazione. 
In effetti, si tratta di un doppio tombeau, i cui piani sovrapposti entrano in relazione attraverso una serrata rete intra- ed intertestuale, poiché quella specie di sopralluogo personale e letterario si combina con un paradossale omaggio parricida di Consolo a tutti gli auctores che egli ha ammirato per poi rinnegarli, come fanno tutti i figli quando intraprendono una crociata contro i propri padri, nella vita e nella letteratura; perché i padri sono stati giudicati troppo spesso «imbell[i], ipocrit[i] o impotent[i]» (Consolo 2000: 89); perché le loro parole non hanno saputo essere l'ultimo baluardo contro il sonno della ragione e il caos.

Sembra, infatti, che quest'ultimo libro, alla stregua di quanto dichiara l'incipit dell'ultimo romanzo di Sciascia, sia un modo per "ancora una volta [...] scandagliare scrupolosamente le possibilità che forse ancora restano alla giustizia» (Sciascia 1989: 7) e, vorremmo aggiungere, alla letteratura. Sennonché tale ricerca ha portato Sciascia a scrivere Una storia semplice mentre, per Consolo, è venuta fuori una storia maledettamente complicata, condotta come un'analisi retrospettiva, alla maniera delle inchieste politico-filologiche dello scrittore di Racalmuto, ma senza ricorrere fino in fondo ai suoi strumenti di investigazione della realtà e del discorso; quelli di Pausania, tanto per intenderci, il saccente geografo che ne L'olivo e l'olivastro sentenziava: "a me è affidato il dovere del racconto: conosco i nessi, la sintassi, le ambiguità, le malizie della prosa, del linguaggio» (Consolo 1999a: 39).

Come il suo protagonista, lo scrittore Gioacchino Martinez, Consolo si è infatti rifiutato di entrare nella logica «fiduciosamente comunicativa, di padre e fratelli - confrères - più anziani, involontari complici, pensav[a], dei responsabili del disastro sociale» (Consolo 2000: 127). Da allora, per lui, ha trovato «solo senso il dire o ridire il male, nel mondo invaso in ogni piega e piaga dal diluvio melmoso e indifferente di parole atone e consunte, con parole antiche o nuove, con diverso accento, di diverso cuore, intelligenza. Dirlo nel greco d'Eschilo, in un volgare vergine come quello di Giacomo o di Cielo o nella lingua pietrosa e aspra d'Acitrezza» (Consolo 1999a: 77). Fino a Lo Spasimo di Palermo. Fino al momento in cui, dopo un ultimo tentativo per lottare contro il disordine, il silenzio sostituisce il coro che, poc'anzi ancora, «in tono alto, poetico, in una lingua non più comunicabile, commenta[va] e lamenta[va] la tragedia senza soluzione, il dolore senza catarsi» (Consolo 1999b: 262).

Come la maggior parte dei libri precedenti di Consolo, Lo Spasimo di Palermo è la storia di un viaggio, quello dello scrittore Gioacchino Martinez. Un viaggio nello spazio, da Parigi a Milano e da Milano a Palermo, e un viaggio nel tempo, quello dell'infanzia durante la Seconda Guerra Mondiale, dei primi trasporti e del matrimonio con Lucia, che diventerà sua moglie e la madre di Mauro, il figlio rifugiatosi appunto a Parigi per sfuggire alla repressione politica della fine degli "anni di piombo", mentre Lucia sprofonderà a poco a poco nella follia per sfuggire all'insopportabile realtà della violenza mafiosa degli anni Ottanta e dei primi anni Novanta, prima di scomparire nel buio. Un viaggio a misura d'uomo dunque, chiuso e concluso, almeno in apparenza, nei limiti di una vita d'uomo, 
con gli inevitabili punti di contatto con il mondo che gli sta attorno, cioè con la Storia con la S maiuscola i cui disordini e le cui convulsioni si ripercuotono sull'esistenza dell'individuo e la possono travolgere senza remissione.

Però, anche se l'invocazione liminare è quella di un Omero-Consolo a un Ulisse-Consolo la cui commistione-confusione sarà nel libro un'ulteriore fonte di ambiguità, non si tratta più, proprio per lo Spasimo, del solito nostos ulissiano di cui L'olivo e l'olivastro è forse l'espressione più compiuta e dichiarata, ma si tratta piuttosto di un percorso di anamnesi dalle coordinate incrociate e sovrapposte, che trasforma l'effettivo ritorno a casa del protagonista e il suo tentativo di ricongiungersi con il proprio passato, in viaggio penitenziale (Francese 2015: 66 e 104; Consolo 1999a: 19). Un viaggio per espiare colpe insite nelle confessate manchevolezze di chi scrive attraverso la di nuovo paradossale, nonché scaramantica, trasformazione della scrittura/lettura di questo libro in una complessa operazione di cifratura e decodifica storico-letteraria.

La principale fonte di complessità sta nella frammentazione o variazione della voce narrante. Il procedimento non è nuovo e Consolo lo ha ampiamente sfruttato fin dal Sorriso e anche in Nottetempo (Consolo 1994). Solo che allora si trattava di dare alle "voci' dei margini» (Consolo 1997: 182) una possibilità di farsi sentire pur riconoscendo l'inadeguatezza della scrittura, dotta per definizione, nella restituzione di un'oralità per così dire primitiva, nonché «l'impotenza, l'incapacità di dire, di raccontare la vita, il patimento» (Consolo 1994: 53) per colui che scrive. Il dilemma sembrava si fosse risolto in senso sperimentalista con l' «adottare [...] moduli stilistici della poesia, riducendo, per rimanere nello spazio letterario, lo spazio comunicativo, logico e dialogico proprio della narrazione» (Consolo 1997: 183); e l'intento di far combaciare logos, epos ed ethos pareva fosse stato raggiunto ne L'olivo e l'olivastro come dimostrano l'omogeneità di tono e la stabilità dell'istanza narrativa (Bossi 2012).

Ma con Lo Spasimo di Palermo si sgretola quel fragile equilibrio nella frantumazione dei punti di vista e nella variazione dei toni e dei gradi della diegesi. Più conturbante ancora: vengono di nuovo sfruttate soluzioni narratologico-espressive da tempo dichiaratamente abbandonate e, nel libro stesso in cui ricompaiono, presentate come detestabili (Consolo 2000: 105).

Il corifeo-narratore de L'olivo e l'olivastro - che cantava la storia del nuovo Ulisse in una specie di discorso indiretto libero intriso di quella tragicità antica che per Consolo è «l'esito ultimo [...] della [sua] ideologia letteraria, l'espressione estrema della [sua] ricerca stilistica» (Consolo 2007: 21) e in cui si intercalavano registrazioni/restituzioni di voci testimoniali - è ancora presente nel prologo in corsivo, ma solo per un ideale passaggio di testimone narrativo ed esistenziale allo stesso Ulisse. E nel suo augurio per il viaggio da compiere sta senz'altro la spiegazione delle contraddizioni e dei paradossi che abbiamo segnalati fin qui: "Ora la calma t'aiuti a ritrovare il nome tuo d'un tempo, il punto di partenza. In my beginning is my end» (Consolo 2000: 9). 
Solo attraverso la volontà di tornare al punto di partenza, geografico ed esistenziale, si può infatti spiegare il metaforico ritorno a una specie di autobiografismo letterario che si palesa attraverso una fitta rete di autocitazioni più o meno esplicite come, ad esempio, quella testuale tratta da Le pietre di Pantalica (Consolo 1988: 166): «Palermo è fetida, infetta. In questo luglio fervido esala odore dolciastro di sangue e gelsomino». Messa in bocca al giudice che ne Lo Spasimo di Palermo (con la S maiuscola) viene omaggiato in quanto uno tra i pochi difensori della polis e del vivere civile, quella citazione già parlava dello spasimo (con la s minuscola) di Palermo e, anche se Gioacchino-Consolo si schermisce dicendo che «sono passati da allora un po' di anni», il giudice - di nuovo sciascianamente, come conferma il paragrafo successivo - profetizza: «Ma nulla è cambiato, creda. Vedrà, il prossimo luglio sarà uguale... o forse peggio» (Consolo 2000: 115).

Allo stesso modo, questo appellarsi di continuo a Sciascia come pure a Manzoni con riferimenti espliciti - oltre che alla peste che allora infettava Milano come ora infetta Palermo - anche al famoso manoscritto manzoniano (Consolo 2000: 106), ossia alla finzione letteraria destinata a conferire verosimiglianza al testo fondatore dei Componimenti misti di storia e d'invenzione, delinea una specie di autobiografia non più o non solo personale, bensì familiare, nel senso di una famiglia spirituale, un omaggio intertestuale in forma di metaromanzo di formazione «ai suoi» (Consolo 2000: 36), come egli chiama i suoi autori prediletti.

Sembra infatti che Consolo si chieda se proprio nel tornare alle origini, alle fonti e, quindi, a quei «linguaggi logici, illuministici», «alla loro serena geometrizzazione» (Consolo 1997: 182) non risieda la possibilità di dire il male e di sanarlo. Ragion per cui egli conserva effettivamente, ad inizio capitolo, quei paragrafi che segnano «l'interruzione del racconto e il cambio di tono della scrittura, il suo alzarsi di tono, svolgersi in forma ritmica, lirico-poetica», che sono per lui «le parti corali o i cantica latini» (Consolo 2007: 35); quei paragrafi che dicono il male come rottura, alla stregua di quel passaggio dalla luce all'ombra che spaventa la Lucia di Gioacchino (Consolo 2000: 65 e 117) come segno precursore di un'irrimediabile condanna alla follia, con «lei distesa nella stanza che urla e che singhiozza, ritorna dall'estrema soglia, dall'insulinico terrore, entra ed esce per la porta sull'abisso, il tempo è fisso nel continuo passaggio, nell'assenza, nel fondo sono le sequenze, i nessi saldi e veri» (Consolo 2000: 45). E quelli sono i nessi, tra afasia e urlo, che, soli, la poesia, il linguaggio poematico, possono dire.

Ma allo stesso tempo Consolo, come Gioacchino, ha la tentazione di riannodare il discorso interrotto, di riprendere il film dell'infanzia, rappresentato metaforicamente dalla storia di Judex, nel punto in cui è stato tagliato, e farlo ripartire con «la sutura, l'acetone sulla memoria, il nastro che s'attacca, lo scintillio dei carboni, il ronzio che riprende, la storia che prosegue e si conclude». Pena però la riscoperta dei «fotogrammi segreti della sua storia, della vita sua oscura e inconclusa, della frana, delle colpe sepolte e obliate» 
(Consolo 2000: 47). In altri termini, sembra che Consolo si chieda, in un libro che insiste sull'interruzione della comunicazione tra padri e figli a causa delle compromissioni e dei tradimenti rimproverati dai figli ai padri, se non si dovrebbe compiere una sutura tra gli uni e gli altri, tra passato e presente, ricorrendo ai pure ripetutamente rinnegati strumenti di Pausania - il geografo che, come si diceva prima, conosce «i nessi, la sintassi, le ambiguità, le malizie della prosa, del linguaggio» (Consolo 1999: 39). Ed è proprio a quel lavoro di sutura personale e letteraria che si accinge Gioacchino, confrontato al falso ordine della sua biblioteca risistemata "cromaticamente" dalle incolte persone di casa:

Giorni trascorsi a rimettere ordine fra i libri. Da questi doveva ricominciare, dalla chiara geografia, dai confini certi, dal conforto loro, per potersi orientare, riprendere la strada. Oltre, non era che mutamento, cancellazione d'ogni segno, realtà infida, landa d'inganno, sviamento. (Consolo 2000: 102)

A partire dai libri della sua biblioteca, egli comincia dunque a ricostruire una mappa letteraria della propria città, uno di quei Cruciverba (Sciascia 1983) che tanto piacevano a Sciascia, come quello dedicato dal racalmutese a Parigi dove egli si recava, come ricorda lo stesso Consolo (Consolo 2016: 124), compiendo un viaggio le cui tappe erano Palermo-Milano e poi Milano-Parigi, ovvero l'esatto rovescio, quasi a specchio, del viaggio raccontato dalla doppia, se non dubbia, figura di Empedocle-Pausania e compiuto dall'ambiguo Ulisse de Lo Spasimo di Palermo. Infatti l'Ulisse consoliano ha avuto bisogno di attingere di nuovo ai valori civili e letterari della Francia illuminista tanto amata da Sciascia. Questo spiega perché, per la prima volta, non lo vediamo approdare fin dall'inizio del libro alla sua Itaca-Sicilia come invece succedeva nei precedenti racconti: è dovuto prima andare a cercare a Parigi, nella città in cui «tutto parla di letteratura» e dove si parla «quella che Leopardi chiamava una lingua 'geometrizzata'» (Consolo 2016: 132), quei pezzi mancanti, quelle colpe sepolte e dimenticate della propria storia, prima di intraprendere il suo nostos espiatorio (Traina 2001: 43).

A quell'Ulisse, costretto quindi a usare quasi simultaneamente due lingue diverse, una poematica e l'altra geometrizzata, viene assegnato il compito di riannodare i fili spezzati per tentare non solo di dire, poeticamente ed espressivamente, ma anche di denunciare, razionalmente e comunicativamente, i mali passati e presenti e la loro diffusione-dilatazione. Infatti essi hanno ormai da tempo varcato lo Stretto, come mostra la cantica dedicata a Milano, che fu «approdo della fuga, quell'asilo della speranza, antitesi al marasma, cerchia del rigore, probità, orgoglio popolare, civile convivenza, magnanimità e umore, tolleranza». Ormai

Illusione infranta, amara realtà, scacco pubblico e privato, castello rovinato, sommerso dalle acque infette, dalla melma dell'olona, dei navigli, giambellino e lambro oppressi dal grigiore, dallo scontento, scala del corrotto melodramma, palazzo della vergogna, duomo del profitto, basilica del fanatismo e dell'intolleranza, banca dell'avventura e dell'assassinio, fiera della sartoria mortuaria, teatro della 
calligrafia, stadio della merce e del messaggio, video dell'idiozia e della volgarità.

(Consolo 2000: 91)

Una Milano reificata attraverso la soppressione di tutte le maiuscole, una Milano al cui quadro orrifico corrisponde, "di qua dal faro", quello di Palermo. Non la Palermo dell'approdo - e siamo già oltre i tre quarti del libro - di UlisseGioacchino-Consolo al porto che fu anche il loro comune punto di partenza, ancora ingentilita dalla memoria dell'esiliato e dal ricordo dei passati splendori:

Nella luce bianca, vaporosa, apparivano il piano di Sant'Erasmo, la foce melmosa dell'Oreto, le palazzate nuove del sacco mafioso, la Flora e le possenti mura, la Porta dei Greci, la passeggiata delle Cattive, gli antichi palazzi dietro nobiliari, le cupole e i campanili delle chiese, il Càssaro Morto e la Porta Felice, Santa Maria della Catena, la conca stagna affollata d'alberi di lussuose barche della Cala. (Consolo 2000: 98)

Ma la Palermo della banalità secolare del male. «L'amata sua odiata» che non nasconde più il proprio vero viso:

Intrigo d'ogni storia, teatro di storture, iniquità, divano di potenti, càssaro dei criadi, villena degli apparati, osterio di fanatismo, tribunale impietoso, stanza della corda, ucciardone della nequizia, kalsa del degrado, cortile della ribellione, spasimo della cancrena, loggia della setta, casaprofessa della tenebra, monreale del mantello bianco. (Consolo 2000: 122)

Oltre alle scelte stilistiche illustrate da questi due cantica speculari, la cui espressività, non sempre compensata dalla comunicatività dei fatti e delle date, costringe il lettore a indovinare situazioni ed epoche, nonché bersagli della denuncia, il cambiamento maggiore portato dall'ambiguità della nuova istanza narrativa poematico-geometrica sembra risiedere nel filtro che una specie di stream of conciousness di stampo quasi gattopardiano impone alla rimemorazione, come pure nello sfasamento che tale rimemorazione provoca tra tempo e spazio ricordati rispetto ai tempi e allo spazio della narrazione e a quelli della Storia, con la conseguenza di appiattire e concentrare tempi ed epoche diverse nello stesso movimento narrativo. Come quando, ad esempio, a partire dal vivido ricordo della peste manzoniana (Consolo 2000: 85) e senza nessuna rottura temporale apparente, il narratore ci parla del «tempo febbrile delle pesti, del colera di Palermo» (Consolo 2000: 113), giocando peraltro sull'ambiguità tra tempo meteorologico e tempo cronologico, in una sovrapposizione allucinata in cui diventa impossibile discernere in quale epoca vivano $\mathrm{i}$ "ciechi vaiolosi storpi appestati d'ogni sorta che dallo Spasimo si recavano ogni giorno per mangiare alla Dogana» (Consolo 2000: 32).

Infatti, se Consolo, fedele alla sua definizione dello scrittore siciliano come uno per cui «il romanzo storico, e in specie in tema risorgimentale [è un] passo obbligato» (Consolo 1997: 181), ci presenta con Lo Spasimo di Palermo «un ulteriore fallimento delle speranze italiane» per cui «[le] speranze di una rinascita crollano di fronte alla pervasività del potere mafioso, e alle sue infiltrazioni nello Stato repubblicano» (De Gregorio 2013), nelle modalità pratiche sembra invece che egli aspiri, da un lato a cancellare e distruggere la rete cronologica, dialogica e 
narrativa di cui necessita la storia per essere detta o per raccontarsi, dichiarando di «aborri[re] il romanzo, questo genere scaduto, corrotto, impraticabile» (Consolo 2000: 105) e, dall'altro, a ristabilire i nessi logici atti a rendere conto dell'orrenda realtà palermitana e italiana di quegli anni.

L'operazione di distruzione non è nuova: Consolo aveva infatti rivendicato che Il sorriso dell'ignoto marinaio, primo opus del ciclo, fosse classificato nella categoria degli antiromanzi storici, in una formulazione peraltro già ambigua poiché, se è antiromanzo, non può essere racconto e, se non è racconto, non può essere storia, per cui non si vede come un antiromanzo possa essere storico. Infatti sembrava degno della quadratura del cerchio il tentativo da allora condotto da Consolo di raccontare una storia e, addirittura, la Storia, che è la Grande Narrazione per eccellenza senza gli strumenti del racconto, col solo spostare il problema della scrittura «dalla comunicazione all'espressione» (Consolo 2007: 35). Questa è probabilmente la ragione dello scarso effetto di tali scelte sulla società civile e del conseguente mea culpa di Gioacchino-Consolo a destinazione dei figli che, alla stregua di Mauro Martinez, «si nega[no] a ogni confidenza, tentativo di racconto, chiarimento» (Consolo 2000: 42), esattamente come lo stesso Gioacchino Martinez confessa di averlo fatto con i propri padri intellettuali e letterari (Consolo 2000: 129).

Quello che è nuovo, invece, e diretta conseguenza di quanto precede, è la costruzione filologico-letteraria in puro stile sciasciano avviata da ConsoloGioacchino a partire da fatti diversi di storia letteraria e civile (Sciascia 1989b), organizzati e ramificati in modo da costituire quello che Sciascia, ancora, chiamava un "teatro della memoria", cioè un sistema di "oggetti eterni [...] che variamente, alternativamente, imprevedibilmente splendono, si eclissano, tornano a splendere e ad eclissarsi - e così via - alla luce della verità» (Sciascia 1979: 231) poiché, come ha scritto lo zio Mauro di Gioacchino nel suo testamento, là si trova, «negli assoluti libri, la verità umana» (Consolo 2000: 73). Infatti, proprio dai libri di botanica e dal giardino dello zio scatta il primo "teatro della memoria" inscenato da Gioacchino, giacché, per lui come per lo zio, il giardino è «luogo platonico, ordine del mondo [...], immagine del giardino interiore, sogno del ritorno, ripristino». Sotto il segno di questo ritorno e di questo ripristino, che però possono anche portare «offesa, patimento» (Consolo 2000: 49), nasceranno gli ulteriori elementi del suo sistema di "oggetti eterni" destinati a comporre una specie di antistorica - perché sorta dall'eterna e quindi atemporale verità letteraria - Storia delle storie: messe insieme, la storia delle tonnare (Consolo 2000: 59), quella del codice di San Martino - di nuovo di sciasciana ascendenza - e quella del Libro intorno alle palme di Abu-Hâtem-esSegestâni (Consolo 2000: 64) tracciano i confini storico-geografici della Sicilia araba; quella poi, già più complessa e ricorrente, della "prigionia in Algeri di Cervantes e [di] quella insieme, d'un poeta [palermitano], dialettale, Antonio Veneziano» (Consolo 2000: 41, 105, 115, 118) disegna i contorni della Sicilia dei tempi dell'occupazione spagnola e conduce a sua volta alla scoperta, nella topographia e historia general de Argel (Consolo 2000: 107), di una parola che 
permetta a Consolo di collegare gli oggetti letterari già messi in risonanza con la più recente storia siciliana e italiana e, a Gioacchino, di seguire uno dei fili più dolorosi della sua personale storia umana. La parola è "marabutto", e il filo che da essa dipana Gioacchino va dal massacro di suo padre e della madre di Lucia nel Marabutto di Rassàlemi per arrivare all'ultima scena del libro, quella dell'esplosione, in cui, mentre scrive a suo figlio, egli scompare insieme al giudice, figlio della sua vicina di casa: una scomparsa preannunciata da una specie di predizione dell'amico fioraio: «Ddiu ti scanza d'amici e nnimici, e di chiddi chi ti manciunu lu pani. Ddiu ti scanza di marabutta» (Consolo 2000: 124). Tutta questa intricata storia di fili e di figli permette inoltre al lettore di ricostruire, pure se a volte faticosamente, i famosi nessi grazie ai quali Gioacchino-Consolo spera di «scrivere di una storia vera [...] fuori da ogni invenzione, finzione letteraria» (Consolo 2000: 105), insomma, fuori dall'aborrita scrittura romanzesca.

Tuttavia intento alla (per lui) illuminante, salvifica e lenificante ricostruzione letteraria e culturale di un passato comune a sé e, a una seppur «devastata» (Consolo 2000: 105) civiltà mediterranea nella quale riaffondare le proprie radici per ripartire verso un futuro riconciliato, Gioacchino non presta abbastanza attenzione ai messaggi sempre più cupi che provengono dall'altro "teatro della memoria", concorrente del primo, che si sta costruendo quasi suo malgrado a partire da altri elementi anch'essi perfettamente collegati: un altro "teatro" che ha le stesse radici del primo, ma si sviluppa in modo subdolo e morboso esattamente come, a partire dallo stesso tronco, quello dell'olivo in cui Ulisse ha intagliato il proprio letto, si possono sviluppare sia l'olivo che l'olivastro. E infatti, dalla sua stessa casa, anzi dal suo stesso letto appunto, «sporcato ignobilmente» (Consolo 2000: 119) ad opera dei nuovi Proci, dei sempre rinnovati Proci della mafia e della politica, seguendo poi quella strada intitolata al musicista Emanuele d'Astorga da cui Gioacchino credeva di poter cominciare a riprendere possesso della sua città inseguendo memorie storiche e civili, proprio da lì ricomincia invece a proliferare la mai sanata peste, il cui nome, le cui immagini, i cui manzoniani echi percorrono tutto il libro al ritmo sempre più serrato di un'allucinata cavalcata mortifera per le strade, i monumenti della città, per poi proseguire, alla stregua del quadro di Raffaello, la sua anti-Odissea «da Palermo, alla Sicilia, al mondo» (Consolo 2000: 112), a celebrare il Trionfo della Morte (Consolo 2000: 117), della Follia (Consolo 2000: 111) e del Caos (Consolo 2000: 117). Il "teatro della memoria", messo in moto dalla vita, ossia dalla vita di d'Astorga, si ramifica generando un'escrescenza cancrenosa che fagocita progressivamente gli "oggetti eterni" positivi che si stavano sviluppando serenamente sull'altro ramo, quello della riconciliazione, fino a mettere in una luce sinistra, di malaugurio, le notizie sulla chiesa di Santa Maria dello Spasimo "per cui Raffaello aveva dipinto La caduta di Cristo sul cammino del Calvario, chiamato qui Lo spasimo di Sicilia», ma chiamato dal suo artefice "sgomento della Vergine e Spasimo del mondo» (Consolo 2000: 112). Come anche la notizia secondo la quale il pezzo più conosciuto di d'Astorga era uno Stabat mater. Quello Stabat mater le cui note, la cui grafia diversa da quella della scrittura, la 
cui espressività che mai la scrittura potrà raggiungere, anche se cerca di farsi pura espressività e puro suono, bucano ad un tratto la pagina di Consolo a sostituire le parole (Consolo 2000: 125), preannunciando sia lo strazio, lo spasimo della madre del giudice di fronte alla morte del figlio in veste di laico redentore di tutte le colpe dei padri, sia «la sconfitta, la dimissione, l'abbandono della penna» (Consolo 2000: 127), confessati da Martinez nella sua lettera al figlio e quindi da Consolo al suo lettore.

Ci si può chiedere se sarebbe stato possibile evitare tale sconfitta, una sconfitta che ha portato Consolo a riconoscere, con la metafora della lettera interrotta, l'impossibilità di un sereno passaggio di testimone tra le generazioni, per poi chiudersi nel silenzio dopo la morte del suo doppio letterario. Ci si può chiedere se, rifiutando i nessi razionali, la comunicazione dialogica propria del romanzo e in particolare la lettura ipotetico-deduttiva propria del romanzo poliziesco che gli avrebbe permesso di collegare gli indizi, pur presenti nel testo, della proliferazione del male ${ }^{3}$ e, limitandosi a scrivere «solo di cultura, storia, vicende curiose del passato, di questa città com'era un tempo» (Consolo 2000: 74), Consolo-Gioacchino non si sia precluso ogni possibilità di analizzare correttamente il presente e di riformarlo. Ci si può chiedere se non sarebbe stato opportuno approfittare di quel «varco che conduceva nel passato, nel racconto, in cui tutto era accaduto, tutto sembrava decifrabile» (Consolo 2000: 69), per scrivere, a dieci anni dalla morte dell'amico Sciascia, una storia finalmente semplice, invece di considerare che la volontà di riannodare i fili della propria storia personale e civile, di riprendere il film della lotta di Judex contro i cattivi laddove si era interrotto era, per finire, «uno sbaglio» (Consolo 2000: 53).

Quello che possiamo dire è che Consolo ci ha provato. Con Lo Spasimo di Palermo è infatti giunto a quanto di più vicino al romanzo - al, per lui, "impossibile romanzo" - fosse lecito spingersi, senza rinunciare completamente alle scelte e agli impegni di tutta una vita, in quanto scrittore e in quanto cittadino. Lo ha fatto cercando di ibridare la sua abituale scrittura poematica con quella geometrica comunicazione presa in prestito dal «castoro ligure; [dal] romano indifferente, [dall'] amaro [suo] amico siciliano», ma anche da quelli che, come loro, «hanno la forza [...] della ragione, la chiarità, la geometria civile dei francesi» (Consolo 2000: 88): Pasolini, Vittorini, Manzoni, e tanti altri, i cui nomi, i cui libri, compongono a poco a poco un superiore "sistema di oggetti eterni", una specie di borgesiano Aleph letterario.

Sennonché, anche quest'ultimo tentativo per dire il male, seppur condotto sotto l'alto patrocinio di sì autorevoli padri, si conclude con la morte di quelli che, almeno per Consolo, sono, erano, gli ultimi difensori dell'umana civiltà, il giudice e l'intellettuale. E con la loro morte non solo scompare la possibilità di una redenzione civile, ma viene anche dimostrata, attraverso quella specie

3 Basterebbe seguire le tracce lasciate dall'uomo dalle scarpe gialle, dal falso ragioniere, dal mafioso delle Falde... 
di suicidio parricida, l'impotenza della letteratura - i cui rappresentanti sono pur stati convocati in schiere compatte - ad arginare, con qualsiasi mezzo o strumento, il dilagare del male.

Il che non ci deve stupire, poiché neanche Judex, che pure «giudica e sentenzia fuori dalle leggi» (Consolo 2000: 129), ci può riuscire: ovunque e comunque «la nera sagoma trionfa del giustiziere» e «si conclude il feuilleton fuori da leggi, tribunali, si scioglie la vendetta precivile nel sentimento, si ricompone l'ordine del denaro e del potere» (Consolo 2000: 47) .

\section{Bibliografia}

Bossi, L., 2012, «'L’olivo e l’olivastro’ de Vincenzo Consolo: pour une Odyssée du désastre», Cahiers d'études italiennes, $\mathrm{n}^{\circ} 14 / 2012$, Les années quatre-vingt et le cas italien, a cura di Barbara Aiosa e Leonardo Casalino, p. 201-212.

Consolo, V., 1988, Le pietre di Pantalica, Milano, Mondadori.

Consolo, V., 1992, Nottetempo, casa per casa, Milano, Mondadori.

Consolo, V., 1997, Il sorriso dell'ignoto marinaio, Milano, Mondadori.

Consolo, V., 1999a, L'olivo e l'olivastro, Milano, Mondadori.

Consolo, V., 1999b, Di qua dal faro, Epigrafi, Milano, Mondadori, «Oscar».

Consolo, V., 2000, Lo Spasimo di Palermo, Milano, Mondadori, «Oscar».

Consolo, V., 2016, Passi a piedi, passi a memoria in Sciascia e Parigi, Catania, Passim Editore.

Consolo, V., 2007, La metrica della memoria, in Vincenzo Consolo, éthique et écriture, Paris, Presses Sorbonne Nouvelle.

De Gregorio, F., 2013, In ricordo di Vincenzo Consolo in: Eidoteca, https:// eidoteca.net/2013/04/30/in-ricordo-di-vincenzo-consolo/

Francese, J., 2015, Vincenzo Consolo: gli anni de «l'Unità» (1992-2012), ovvero la poetica della colpa-espiazione, Firenze University Press.

Sciascia, L., 1979, Nero su nero, Torino, Einaudi.

Sciascia, L., 1983, Cruciverba, Torino, Einaudi.

Sciascia, L., 1989, Una storia semplice, Milano, Adelphi.

Sciascia, L., 1989b, Fatti diversi di storia letteraria e civile, Palermo, Sellerio.

Traina, G., 2001, Vincenzo Consolo, Firenze, Cadmo.

4 «Questo di oggi è un libro sul presente, ma anche un ripercorrere gli anni Sessanta, con il terrorismo vissuto a Milano e anche con quello che è il male della Sicilia, con le atrocità delle stragi di mafia. Infatti, il libro si conclude con la strage di Via d'Amelio e con la morte del giudice Borsellino. Questo è stato il mio progetto letterario. Poi, naturalmente, accanto a questi libri, ci sono dei libri collaterali, ma tutti convergono su questa idea, cioè, sul potere, sulla corruzione del potere» in Intervista inedita a Vincenzo Consolo, a cura di Irene Romera Pintor, http://vincenzoconsolo.it/?p=1299 poi pubblicata in Vincenzo Consolo, "Autobiografia della lingua" - Conversazione con Irene Romera Pintor http://books.openedition.org/res/318. 